of support varies depending on specialty and setting. For doctors who have worked in a setting where the minimum emergency response includes a Resuscitation team, moving to an environment with less support available is a challenge.

In our unit, the protocol following an urgent call is for the on-call doctor (who has access to basic resuscitation equipment) to attend and assess the need for paramedics and transfer to local hospital. Stress can be worsened by change of environment, change of expectation and concern about best management in new settings.

Method. A cohort of junior doctors were recruited. Baseline assessment included rating their confidence level (scale 1- 10), listing common medical and psychiatric scenarios they had experienced and those they felt least confident managing. Over a period of 10 weeks, follow-up data was obtained. Interventions to improve confidence were assessed during this period, including a handbook and a teaching session on emergency medications. At the end of the project a wordcloud was created in response to the request to "choose 5 words to describe your feelings when called to an emergency". Identified themes have been fed back to relevant senior staff and will form the basis of future projects.

Result. The initial average confidence score improved from 4.9 to 9.2 and was sustained out to 14 weeks. According the word cloud the most commonly used words were "morale" and "education". Conclusion. Prior to the study, confidence levels amongst the Junior Doctors was low. Introduction of the handbook and teaching session led to an improvement which was sustained. Key themes identified using a word cloud were "morale" and "education".

For junior doctors moving from between services, different expectations and protocol for management of emergencies can influence confidence levels. Psychiatric units should be cognisant of these concerns and implement evidence-based intervention to support junior doctor confidence and improve quality of working experience.

Perception of junior doctors about local induction in Birmingham and Solihull Mental Health Foundation Trust - A quality improvement project

Saima Jehanzeb ${ }^{1 \star}$, Kozara Nader ${ }^{2}$ and Ruth Scally ${ }^{2}$

${ }^{1}$ Birmingham and Solihull Mental Health Foundation Trust and

${ }^{2}$ Birmingham and Solihull Mental Health NHS Foundation Trust

${ }^{\star}$ Corresponding author.

doi: 10.1192/bjo.2021.532

Aims. A quality improvement project was undertaken to understand the perception of trainees about the quality of the local induction delivered by Birmingham and Solihull Mental Health Foundation Trust (BSMHFT). The primary aim was to evaluate the current local induction programme, following concerns raised by previous trainees in National Training Survey (General Medical Council) and local inspection. Our secondary aim was to devise a revised induction programme based on the trainees' identified needs.

Method. Two anonymised questionnaire surveys were emailed to all Foundation Year Trainees, Core Psychiatry Trainees and General Practice Speciality Trainees working in BSMHFT, in December 2019 and March 2020, using trust survey monkey.

Result. The overall response to survey was 60 percent. 44.44 percent of the responses came from Core Psychiatry Trainees, with
27.78 percent responses each from Foundation Year Trainees and GP Speciality Trainees. Local induction was defined as induction specific to place of work (47.06\%), trust based induction (41.18\%) or all of the above options (11.76\%) by trainees. $83.33 \%$ of all trainees had received local induction, whereas $16.67 \%$ did not have any local induction at the start of their post. $11.12 \%$ trainees were very satisfied and $44.44 \%$ were satisfied with local induction. 72.22 percent of the trainees were informed about of the local induction, prior to starting the post.

$33.3 \%$ trainees had a paper version, $22.22 \%$ had an electronic version of local induction pack, whereas $44.44 \%$ had no induction pack. $55.55 \%$ of those trainees who had an induction pack, $43.75 \%$ found it very helpful and $56.25 \%$ did not find it helpful.88.89\% thought having a local induction would be helpful, whereas 11.11 percent did not feel it would help. $94.44 \%$ of the trainees completed a local orientation checklist with their consultants. Some of the trainees experienced difficulty in gaining access to electronic prescribing, electronic patient record system (RIO), and identity badges (ID) at the beginning of their post.

Conclusion. $11.12 \%$ trainees were very satisfied, $44.44 \%$ were satisfied, $22.22 \%$ were neither satisfied nor dissatisfied and $22 \%$ were dissatisfied, with local induction. $88.89 \%$ of the trainees thought having a local induction pack would be helpful. Based on the trainees identified needs we developed a template for local induction pack for each post. Clinical supervisors have agreed to take the lead in preparing the local induction pack specific to their post with trainees.We aim to repeat the survey after implementing the changes identified by trainees based on their training needs.

\section{Planning effective mental healthcare in prisons: findings from a national consultation on the care programme approach in prisons}

Jemini Jethwa* and Kate Townsend

The Royal College of Psychiatrists

${ }^{*}$ Corresponding author.

doi: $10.1192 /$ bjo.2021.533

Aims. The Care Programme Approach (CPA) can be an effective tool in coordinating the care and treatment needs of people with mental illness and learning disabilities. Within prisons settings, the CPA has been poorly implemented and the principles underpinning this approach have been lost. The aim of this research was to look at the key themes identified as part of a consultation process to develop quality guidance on planning effective mental healthcare in prisons in relation to the CPA.

Method. The consultation exercises included telephone interviews and hosting a national consultation event to represent the views of prisons nationally. It was conducted by the Quality Network for Prison Mental Health Services, a quality improvement initiative organised by the Royal College of Psychiatrists' Centre for Quality Improvement.

Result. The results derived from the consultation process indicates that $\mathrm{CPA}$ in prisons is inconsistently adopted and that there is lack of confidence in the process from prison mental health teams, particularly with how to engage community mental health teams.

Conclusion. This concludes that there is a substantial need for standardisation and consistency in the application of the CPA process within prisons, for the purposes of enhanced care delivery, greater continuity of care, and improved patient outcomes. 